\title{
LOCALIZADORES APICALES EN ENDODONCIA
}

${ }^{1}$ Monique Marie Gay Ortiz, ${ }^{2}$ Oscar Gustavo Serrano Navas

${ }^{1}$ Odontóloga U Javeriana, Residente II Año Posgrado de Endodoncia, U. Santo Tomás.

${ }^{2}$ Odontólogo U. Santo Tomás, Residente II Año Posgrado de Endodoncia, U. Santo Tomás.

\author{
Autor responsable: Monique Marie Gay $O$ \\ e-mail:mmgto@ustabuca.edu.co
}

\section{RESUMEN}

Determinar la longitud de trabajo es de vital importancia durante la realización de una endodoncia, ya que limita la preparación bioquimiomecánica y posterior obturación del sistema de conductos radicular. Existen diferentes métodos para la localización de la constricción apical o CDC, dentro de los cuales se encuentra el método táctil, el método radiográfico y los aparatos electrónicos conocidos como localizadores apicales. Estos funcionan utilizando tres principios básicos: la resistencia eléctrica, la frecuencia o impedancia y el promedio de varias frecuencias, siendo este último, el que proporciona los resultados más precisos y permite localizar la constricción apical en conductos húmedos por sangre, fluidos, sustancias electrolíticas, como el hipoclorito de sodio y el tejido pulpar vital o necrótico. Aunque, este método disminuye el tiempo de trabajo, no sustituye el uso de las radiografias; por el contrario, se complementan y aumentan el número de éxitos en los tratamientos de conductos radiculares. [Gay MM, Serrano OG. Localizadores apicales en endodoncia. Ustasalud 2003;1:33-41].

PALABRAS Cl.AVES: Localizador apical, Constricción cemento - dentina - conducto (CDC), Constricción apical, Resistencia eléctrica.

\section{Apex Locator in Root Canal Treatment}

\begin{abstract}
During the accomplishment of a root canal treatment is the vital importance to determine the work length, since it limits the extention of the cleaning, shaping and later filling of the root canal system. There are different methods for the location from the apical constriction or Cemento-Dentino-Canal junction (CDC): the tactile method, the radiographic and electronic methods, like apical locators, that work using three basic principles. Those principles are the electrical resistance, the frequency or impedance and the average of several frequencies, being the last one, the one that provides the precise results and allow to locate the apical constriction in the presence of moisture, blood, fluids, electrolytic substances like the sodium hypochlorite and vital or non vital pulp. Although this method diminishes the working time, does not replace the use of the x-rays. It complement and increase to the number of successes in roots canals therapy.
\end{abstract}

KEY WORDS: Apex locator, Cemento-Dentino-Canal junction (CDC), Apical constriction, Electrical resistance.

Recibido para publicación: marzo 4 de 2003, Aceptado para publicación: abril 30 de 2003.

\section{INTRODUCCIÓN}

Determinar la longitud de trabajo o conductometría es de vital importancia durante la realización de una endodoncia, ya que limita la extensión de la preparación bioquimiomecánica y posterior obturación del sistema de conductos radicular, permite obtener resultados favorables en el tratamiento y evita la aparición de molestias postoperatorias.
La constricción apical localizada en cemento y/o dentina, corresponde al punto más estrecho y con menor riego sanguíneo en el interior del conducto, A nivel biológico la constricción apical o CDC (Constricción Cemento - Dentina - Conducto) se constituye como el accidente morfológico más relevante en cuanto a la longitud de trabajo se refiere, porque permite la adecuada conformación de un tope para la condensación del material de 
obturación, favorece el selle apical y permite el control del proceso inflamatorio gracias a la presencia del riego sanguíneo funcional en la zona apical inmediatamente adyacente a ésta; adicionalmente, la gran mayoría de conductos laterales y accesorios se encuentran en este nivel. ${ }^{1}$

La constricción apical, generalmente coincide con la zona de transición entre la dentina y el cemento. Un tratamiento de endodoncia se realiza a nivel del complejo dentino-pulpar, cuyo objetivo es tratar el conducto radicular formado por dentina, para que los cementoblastos, que recubren el cemento, contribuyan a un cierre apical biológico, objetivo final del tratamiento. ${ }^{1}$ (Figura 1)

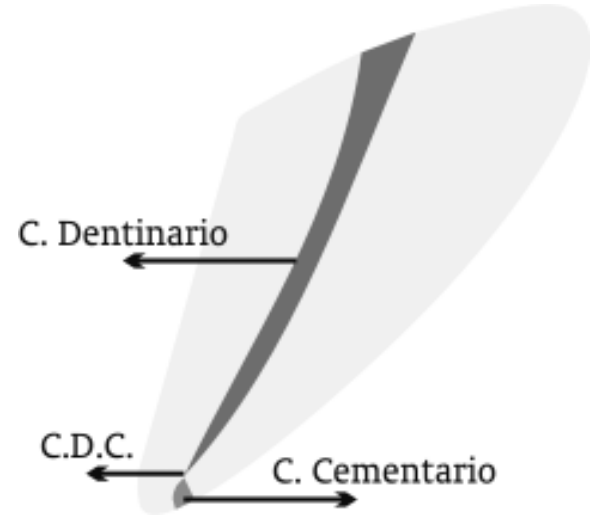

Figura 1. Esquema de la ubicación del CDC en un conducto radicular.

Debido a que es imposible visualizar directamente la CDC, determinar la longitud del conducto radicular requiere una cuidadosa valoración.

Durante mucho tiempo se ha utilizado el método radiográfico en el cual el límite apical corresponde al ápice radiográfico (borde de la raíz en las radiografias) y es según Shilder, la única referencia que puede ser identificada sin ninguna falla durante la limpieza y conformación de conductos; ${ }^{1}$ pero su principal desventaja es que no detecta la constricción apical y sólo se puede observar con exactitud el ápice radiográfico, que se encuentra entre 0,5 y $1,5 \mathrm{~mm}$ de la CDC. Por esto se iniciaron nuevas investigaciones con el fin de crear aditamentos que permitan suplir estas deficiencias. A partir de ahí surgieron los primeros localizadores apicales, que fueron desarrollados hace más de 30 años; con el tiempo han evolucionado hasta llegar a ser aparatos muy útiles y precisos en la práctica endodóntica, ya que permiten determinar la constricción apical cuando son utilizados correctamente y se tiene experiencia de su manejo. ${ }^{2}$

\section{MÉTODOS PARA DETERMINAR LA LONGITUD DEL CONDUCTO}

Existen varios métodos para determinar la longitud del conducto radicular. Kuttler, nombra 5 métodos dentro de los cuales se encuentran: el método táctil, el de rejillas milimétricas en radiografías, las radiografias y cálculos matemáticos, los aparatos electrónicos y el tanteo; también están el método con punta de papel y la respuesta dolorosa del paciente. ${ }^{1-3}$

El método radiográfico es el más utilizado para determinar la longitud del conducto radicular. ${ }^{1,4}$

En 1996, Prattern y colaboradores demostraron que el promedio de desviación de la constricción apical para el localizador apical Endex coincidió en un $59 \%$ de los dientes estudiados $(n=27)$, siendo significativamente menor $(\mathrm{p}<0.05)$ que el promedio obtenido con el método radiográfico, en el que sólo el $18 \%$ de los dientes $(n=5)$ estudiados coincidieron con la constricción apical, 63\% ( $\mathrm{n}=17$ ) estuvieron cortos (menos de $0.5 \mathrm{~mm}$ ) y $30 \%$ ( $\mathrm{n}=$ 8) estuvieron largos (más de $0.5 \mathrm{~mm}$ ); se puede deducir que el método radiográfico tiende a subestimar la longitud de trabajo. ${ }^{4}$

El uso de radiografías, para la localización de la constricción apical, presenta ciertos inconvenientes, debido a que se obtiene una imagen bidimensional de un objeto que, en realidad, es tridimensional; además de las limitaciones anatómicas, como la densidad ósea, la superposición de imágenes y anomalías de posición del foramen en el conducto radicular, entre otras, sin olvidar los riesgos de la radiación. ${ }^{5}$

Pero los dos principales inconvenientes son: en primer lugar, que no detecta la constricción apical y sólo se puede observar con exactitud el ápice radiográfico $0^{1-4} \mathrm{y}$ en segundo lugar, obliga a usar limas de un diámetro determinado para permitir su correcta visualización (es difícil observar con claridad limas con un diámetro inferior a $15 \mathrm{~mm})^{2}$ 
Por último, está el método electrónico o los localizadores apicales, aditamentos que permiten determinar la longitud de trabajo, cuando son utilizados correctamente y se adquiere experiencia en su manejo. Se basan en la diferencia entre la carga eléctrica de los tejidos del ligamento periodontal y cualquier punto en el interior del conducto. Una de las ventajas es reducir el número de radiografias durante el tratamiento. ${ }^{2}$

\section{PRINCIPIO DE FUNCIONAMIENTO DE LOS LOCALIZADORES APICALES}

Cuando a un circuito eléctrico se le incorpora una resistencia no óhmica la interferencia al paso de electrones se conoce como impedancia; bajo este principio trabaja la última generación de localizadores apicales.

Al disminuir la distancia en relación con la CDC también lo hace el valor de impedancia, y a medida que esto sucede se va acercando al valor de impedancia de la CDC o del ligamento periodontal. ${ }^{-9}$

Los localizadores funcionan formando un circuito eléctrico (Figura 2), que consta de un aparato que controla el voltaje (V) al que se le agrega la lima, que se comporta como un conductor de electricidad; la lima entra en el diente, la resistencia, está dada por el material orgánico que se encuentra dentro del conducto. El aparato lo que busca es medir la resistencia entre la punta de la lima y el foramen apical o CDC.

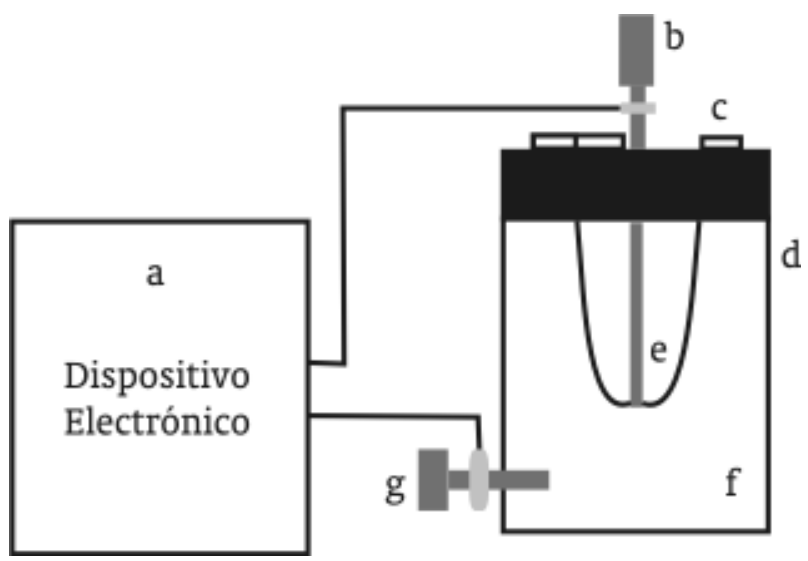

Figura 2. Modelo Experimental de Localizador Apical (a) Dispositivo electrónico: analizador de impedancia. (b) Lima profile \# 1. (c) Resina de auto curado. (d) Recipiente. (e) Diente. (f) Solución salina. (g) Tornillo de acero inoxidable.
Para que esto sea posible se coloca un clip labial, cuya función es cerrar el circuito con el foramen; de esta manera la punta de la lima entra en el conducto, encuentra tejido, la corriente que viene por la lima pasa a través del tejido y sale por el foramen cerrando el circuito en el clip.

\section{EVOLUCIÓN HISTÓRICA}

Si bien los localizadores apicales han sido incorporados recientemente a los recursos de los especialistas en endodoncia, no es un dispositivo nuevo, puesto que fue desarrollado desde el año de 1942, por Suzuki, quien descubrió que la resistencia eléctrica entre un instrumento insertado en un conducto radicular y un electrodo unido a la mucosa oral tiene un valor constante, y detectaba un cambio en la resistencia del conducto con una sola fuente. ${ }^{7,10-12}$

En 1962, Sunada utilizó un dispositivo de corriente directa en un circuito simple y encontró la constante de la resistencia eléctrica para el ligamento periodontal y la membrana mucosa con un valor de $(6,5 \mathrm{kO}))^{1,4,7,8,13-15}$ Posteriormente, en 1969, se fabricó el Canal Meter (Onuki Co Médicos); Este sistema utilizaba una corriente alterna de $150 \mathrm{~Hz}$, que causaba dolor al paciente; por lo que en 1971, se realizó un ajuste en la frecuencia, que permitió el desarrollo del Endodontic Meter. ${ }^{7}$ El Sono-Explorer (Hayashi Dental suplí, Tokio, Japan) desarrollado por Inoue, es calibrado en el surco periodontal de cada diente. ${ }^{7}$ Hasegawa en 1979 presentó el Endocater (Yamaura Seisokushu) que utilizaba una onda de alta frecuencia (400 kilociclos) como localizador apical, con un electrodo conectado a la lima y otro a la silla, realizando una medida correcta en presencia de líquidos conductores, siempre y cuando se utilizara una lima con un forro especial, que funcionaba como aislante; sin embargo, no funcionaba en conductos estrechos. 5,7,16

En 1989 es introducido al mercado el localizador apical Apit, que mide la diferencia entre frecuencias, proporciona una medida exacta de un conducto radicular, incluso en presencia de un electrolito dentro de éste. Sin embargo, debe ser calibrado para cada conducto individualmente en una misma raíz. ${ }^{7} 13$

Kobayashi, en 1991 desarrolló el método del cociente. Este método mide simultáneamente la impedancia de dos frecuencias diferentes en un mismo circuito; el cociente 
de las impedancias se calcula y se expresa en los términos de la posición del electrodo de la lima dentro del conducto. ${ }^{7-9}$

\section{TIPOS DE LOCALIZADORES APICALES}

Los localizadores funcionan según tres principios básicos que miden la longitud de los conductos radiculares, usan la resistencia, la frecuencia o la impedancia y el promedio de varias frecuencias. ${ }^{2,17}$

\section{LOCALIZADORES TIPO RESISTENCIA}

Los localizadores de Primera Generación o de tipo resistencia se basan en la teoría de la resistencia eléctrica desarrollada por Sunada, en 1962. . $, 4,7,8,13-15$ El principio en que se fundamenta es que la resistencia eléctrica entre el periodonto y la membrana mucosa registra valores constantes, en cualquier lugar del periodonto, sin importar la edad del paciente, la forma o tipo de diente y utiliza una corriente directa. ${ }^{1} \mathrm{Al}$ hacer avanzar la lima a través del conducto, ésta entra en contacto con el tejido periodontal apical; entonces la resistencia eléctrica del localizador apical y la lima y la mucosa bucal son iguales. El aparato indica que la lima alcanzó el ápice.

Con este sistema, los conductos debían estar secos, debido a que en presencia de soluciones electrolíticas, como el hipoclorito de sodio, se alteraba la conducción eléctrica, por lo tanto, la medición era errada.

Algunos de los localizadores de primera generación son el Root Canal Meter y el Endodontic Meter (Onuki Medical Co.), el Sono Explorer Mark I, II, III, (Hayashi Dental Supply), el Neosono-MC (Amadent, Cherry Hill, NJ, USA)

El Neosono-MC (Amadent, Cherry Hill, NJ, USA) utiliza una micropuntadora para señalar errores, es automático, no requiere calibración y genera lecturas confiables incluso en presencia de electrolitos, como el hipoclorito de sodio. La principal ventaja de este localizador es que se requiere un conducto relativamente seco; si no lo está, las lecturas tienden a ser cortas, en relación con el foramen apical. ${ }^{2}$

\section{LOCALIZADORES TIPO IMPEDANCIA (UNA FRECUENCIA)}

Los localizadores de Segunda Generación o tipo impedancia hicieron su aparición en los años ochenta; su principio se basa en la detección y el decremento súbito de ésta en la constricción. Su ventaja, con respecto a los de la Primera Generación, es que miden conductos húmedos gracias a un capuchón de plástico colocado en unas sondas especiales; sin embargo, éste se deterioraba por su frecuente uso y procedimientos de esterilización haciendo que se trabara en la entrada del conducto. Un ejemplo es el Endocarter (Hygenic Corp, Akron OH) 1979. ${ }^{1,}$ 3, 5,16

Keller, Brown y Newton, evaluaron la exactitud del Endocater (Hygenic Corp, Akron OH), comparándolo con los ajustes realizados en las radiografías, por recomendación de un endodoncista experimentado. Utilizaron un total de 99 conductos de dientes anteriores y posteriores. Los resultados indicaron que el evaluador realizó una medida adecuada con la lima de $+/-1 \mathrm{~mm}$ del CDC en el 95.8\% de los casos, mientras que con el Endocater I fue de $67.7 \%$. El 34.4\% ( $\mathrm{n}=33$ ) de los casos con el localizador electrónico fueron clínicamente aceptables y no requirieron ningún ajuste por parte del evaluador. Se observó una diferencia estadísticamente significativa $(\mathrm{p}<0.003)$ entre la capacidad del evaluador para localizar la CDC y la capacidad del Endocater. El 59.6\% ( $n=59)$ de la longitud de trabajo determinada electrónicamente se encontraba más allá del $\mathrm{CDC}$, mientras que en los conductos ajustados por el operador, sólo se presentó en 31 de los 99 conductos (32.3\%). ${ }^{16}$

\section{LOCALIZADORES TIPO FRECUENCIA}

En los años noventa surgió la Tercera Generación o de doble frecuencia, que miden la impedancia en dos frecuencias eléctricas distintas. Están el Endex (Osada Electric Co., L.A, CA) 1989, Justy II (Yoshida Co., Japan) y el Root ZX (J. Morita Corp., Tustin, CA) 1991.

El principio de funcionamiento del Endex es medir la diferencia máxima de impedancia entre los electrodos. Requiere una calibración simple para cada paciente, administra un voltaje muy bajo, reduce la respuesta dolorosa del paciente y es relativamente fácil de utilizar. Su principal ventaja es que puede ser utilizado en conductos húmedos por sangre, fluidos, hipoclorito de sodio y tejido pulpar. 
En un estudio in vivo del Endex, se encontró que no era afectado por la pulpa dentro del conducto, o por hemorragia, exudado o hipoclorito de sodio. Esto sugiere que antes de medir la longitud de trabajo es ideal humedecer el conducto, para favorecer la conductividad eléctrica. ${ }^{13}$

La eficacia para determinar la longitud de trabajo del Endex (Osada Electric Co. L.A, CA) fue del 84.56\% ( $\mathrm{n}=37$ ); su uso no presenta variaciones intra e inter operatorias. Además permite la reproducibilidad de los resultados. En este estudio la determinación radiográfica tuvo una eficacia del $97.06 \%$. Sin embargo, este resultado puede variar, ya que se trata de una técnica no reproducible y subjetiva. Estas variaciones son estadísticamente significativas $(\mathrm{p}<0.05){ }^{6}$

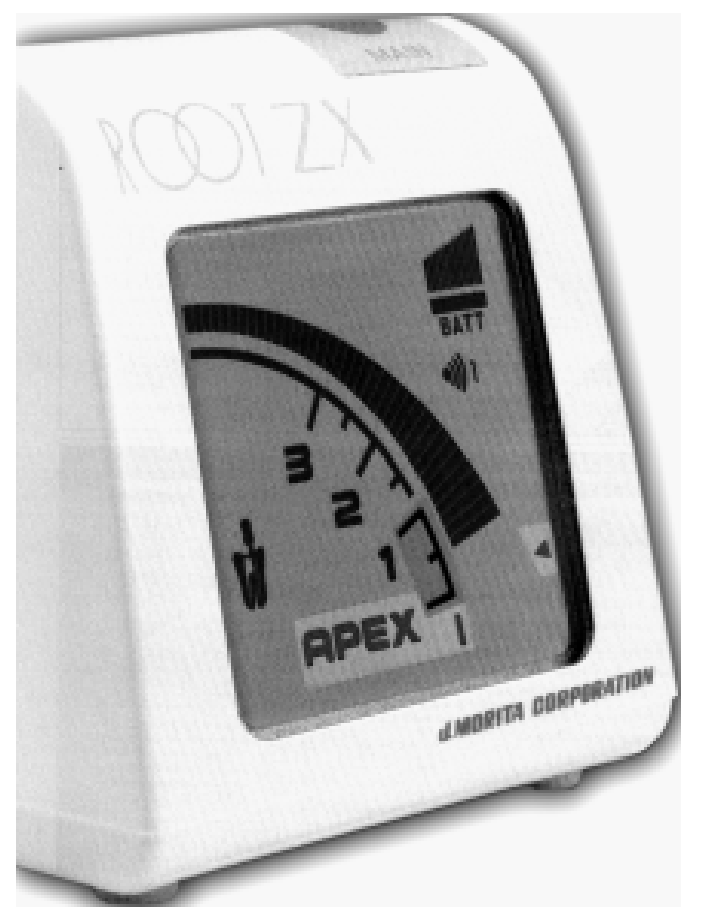

Figura 3. Localizador apical ROOT ZX (J Morita Co., Tustin, CA). Imagen comercial. Journal of Endodontics Vol. 28 № 11, Nov. de 2002

Kobayashi y col., crearon un nuevo aparato eléctrico, tipo radio (Root ZX, J Morita Co., Tustin, CA) (Figura 3), para medir la longitud del conducto cuando hay electrolitos fuertes dentro de éste. ${ }^{7,8} \mathrm{El}$ localizador mide simultáneamente dos impedancias del conducto, se usan dos fuentes de corriente con dos diferentes frecuencias; luego se calcula el promedio de estos dos potenciales eléctricos proporcionales para cada impedancia. El cociente mostrado en el aparato representa la posición de la punta de la lima dentro del conducto. El Root ZX no requiere ninguna calibración; puede ser utilizado en con- ductos secos o húmedos por electrólitos, como sangre e hipoclorito. El diámetro de la lima no produce ninguna alteración durante la toma de la longitud del conducto.

Actualmente se encuentra en el Mercado un nuevo localizador apical, el Apex Finder, Modelo 7005 (Analitic Endodontic, Orange, $\mathrm{CA}$ ) que maneja cinco frecuencias (0.5 Khz., 1, 2, 4, y 8 Khz.).

En un estudio reciente se realizó una comparación in vivo, utilizando el Apex Zinder, modelo 7005, para determinar la longitud de trabajo en conductos vitales, necróticos y retratamientos en presencia de diferentes electrolitos como sangre, exudado e hipoclorito de sodio. Este localizador apical tuvo una precisión del 86\% para localizar la CDC y presentó una diferencia estadísticamente significativa ( $\mathrm{p}<0.05)$, al ser utilizado en conductos vitales (93.9\%), en conductos necróticos (76.6 $\%)$, y en casos de retratamiento (68.4\%). ${ }^{18}$

\section{CARACTERÍSTICAS DE LOS LOCALIZADORES}

Las principales características por las cuales los localizadores no registran una adecuada medición del conducto, son las caries extensas o las destrucciones que comunican el conducto con la encía, ya que la saliva cierra el circuito, emitiéndose un sonido continuo. Lo mismo sucede cuando hay hemorragia que desborde a la corona. La solución, en el primer caso, es realizar una restauración de la caries o la obturación defectuosa y en el segundo, detener la hemorragia. El localizador también se interfiere con obturaciones, muñones y coronas metálicas; por eso se debe evitar que entre en contacto con metal tanto el gancho labial como la lima (separándola con el dedo o secando la cámara con un algodón).

En raíces largas, con sustancias electrolíticas, la tendencia es dar longitudes de trabajo cortas; para solucionar esto se puede secar el conducto con puntas de papel.

La casa fabricante advierte que el funcionamiento de estos dispositivos se puede afectar por la presencia de calcificaciones y obstrucciones dentro del conducto.

Se realizó un estudio in vitro con el Root ZX para determinar qué efecto tendría la eliminación de las interferencias cervicales y la instrumentación previa para facilitar el paso de las limas a través de los conductos radiculares, hasta alcanzar el foramen apical. Se utilizaron un total 
de 32 conductos, divididos en dos grupos. El grupo 1, o grupo control, no fue manipulado antes del uso del localizador apical Root Zx; el grupo 2 fue instrumentado antes de usar el localizador. Los resultados sugieren que la instrumentación de los conductos radiculares, antes de utilizar el localizador, permite a las limas alcanzar el foramen apical de manera más constante ( $\mathrm{p}$.015), mejorando el funcionamiento del localizador apical Root ZX. ${ }^{11}$

Estudios previos sobre localizadores apicales muestran resultados poco precisos, en presencia de fluidos; sin embargo, el uso de sustancias irrigantes presenta grandes ventajas, como acción antibacteriana y disolución de tejidos, que permite la adecuada limpieza del conducto radicular. ${ }^{12}$

Las soluciones irrigadoras no conductivas permiten detectar mejor la posición de la lima en relación con el foramen, además de interferir menos con las restauraciones metálicas, por orden de mayor precisión distinguen: alcohol, SSN al 0,9\%, EDTA y por último el Hipoclorito de Sodio al $5,25 \%{ }^{19}$

La literatura y algunas casas fabricantes registran que la eficacia del Root ZX no se ve afectada en presencia de distintos líquidos dentro del conducto. Jenkins y colaboradores, ${ }^{12}$ realizaron un estudio para probar la eficacia del localizador Root ZX en presencia de una gran variedad de irrigantes comúnmente utilizados durante el tratamiento de endodoncia. Utilizaron: cloruro de sodio al $0.9 \%$, diluido en agua, lidocaína al 2\% con 1:100.000 de epinefrina, hipoclorito de sodio al 5.25\%, RC prep., EDTA líquido, peróxido de hidrógeno al 3\% y peridex. Los resultados mostraron que la determinación de la longitud no se ve afectada al usar el localizador apical Root ZX con cualquier tipo de solución irrigante, al ser comparada con la longitud real del conducto. ${ }^{12}$

La eficacia del Root ZX, con el uso de hipoclorito de sodio, en diferentes concentraciones $5.25 \%, 2.65 \%, 1.00 \% \mathrm{y}$ $0.50 \%$, sobre modelos de alginato, mostró que no hay diferencias significativas $(p>0.95)$ con el uso de diferentes concentraciones de hipoclorito al medir la distancia entre la punta de la lima y la constricción apical. ${ }^{20}$

No se recomienda su uso en conductos no permeables (calcificados o con material de obturación), fracturas radiculares y en personas con marcapasos (por la posibilidad de interferencias). En estos casos es recomendable consultar un cardiólogo. ${ }^{21}$
En dientes con reabsorciones apicales, con o sin fistula, se recomienda medir con limas de un diámetro mayor. En los casos de retratamiento, el conducto debe estar permeable para poder iniciar el proceso de medición. ${ }^{1}$ Los estudios realizados por Shabahang, en 1996, para establecer la precisión de los localizadores indican que la generación actual posee entre el 75 al 96\% de precisión. ${ }^{15}$

Un estudio in vitro, comparó el Root ZX y el Apit, para localizar la constricción apical en presencia de varios irrigantes. Se utilizaron 41 dientes que fueron irrigados con hipoclorito de sodio al $1 \%$, agua oxigenada al $3 \%$ y cloruro de sodio al $0.9 \%$ como grupo control. Se observó que el Root ZX es más preciso (76-85\%) para medir la longitud de trabajo tanto en la lectura a 0,5 y en "APEX" y sobre todo si el conducto contiene hipoclorito de sodio. El Apit es más confiable en las lecturas que realiza como Apex. Se ve más influenciado por el contenido del conducto, dando medidas más cortas. ${ }^{22}$

Después de la exodoncia y basados en la confirmación visual de la relación entre la punta de la lima y el foramen apical por medio del Estereomicroscopio y/o Microscopio Electrónico de Barrido (SEM), los estudios advierten la tendencia a la sobre extensión en relación con el borde del foramen. Por lo tanto, se recomienda retirar $0,5 \mathrm{~mm} 0$ más en aquellos casos en los que la punta de la lima se localiza en el foramen; y de $1 \mathrm{~mm}$ o más, en los casos en los que la lima se localiza más allá de éste. Con esto se evita la sobre instrumentación y la potencial destrucción de la constricción apical, La longitud de ajuste está sujeta a la corroboración radiográfica. ${ }^{15,23}$

La doble conductometría con localizadores y radiografías aumenta el número de éxitos en los tratamientos radiculares. ${ }^{4}$

\section{VENTAJAS Y DESVENTAJAS DE LOS LOCALIZADORES APICALES ACTUALES}

\section{Ventajas:}

La medición es más exacta y confiable que con los métodos radiográficos. Se han reportado estudios que llegan a $96.2 \%$ de exactitud clínica. Es un procedimiento rápido, fácil y disminuye la cantidad de radiación del paciente y personal médico; permite detectar las perforaciones del conducto; es un método para encontrar el foramen fisiológico y no sólo el ápice radiográfico, con limas de cualquier diámetro; efectúan mediciones con conductos 
húmedos no siendo necesario eliminar el contenido total del conducto; son de mejor calidad y exactitud, fácil uso y adquisición en relación con los anteriores aparatos y con el equipo de $\mathrm{Rx}$, y permiten determinar el nivel de las fracturas horizontales. ${ }^{3}$

\section{Desventajas:}

Se requiere un aparato especial. No se aconseja utilizarlo en pacientes con marcapasos, por la posibilidad de interferencias, aunque no se han reportado accidentes con su uso. ${ }^{21}$ Aunque es posible su uso en conductos húmedos, no es recomendable que la cavidad pulpar esté inundada con la solución irrigante, con sangre o con otros líquidos; su uso es limitado en conductos parcialmente calcificados o con coronas y núcleos metálicos; la lectura en dientes con ápice abierto es generalmente falsa, y, por último, en dientes vitales sus resultados pueden ser inconsistentes. ${ }^{3}$

\section{ÚLTIMOS AVANCES EN TECNOLOGÍA DE LOCALIZADORES APICALES}

El objetivo es mejorar las características de los localizadores apicales, a fin de facilitar al operador su trabajo, así como la búsqueda de una mayor presición, mediante la incorporación de nuevos instrumentos para aumentar o mejorar su eficacia.

Se encuentran el Endex plus (Osada Electric Co.) (Figura 4) y el Apit 7 (modelo EM-S7 Osada) . Estos sistemas incorporan dos funciones, una automática y otra manual; dependiendo de la técnica usada o de las condiciones especiales que se puedan encontrar, se utiliza uno u otro.

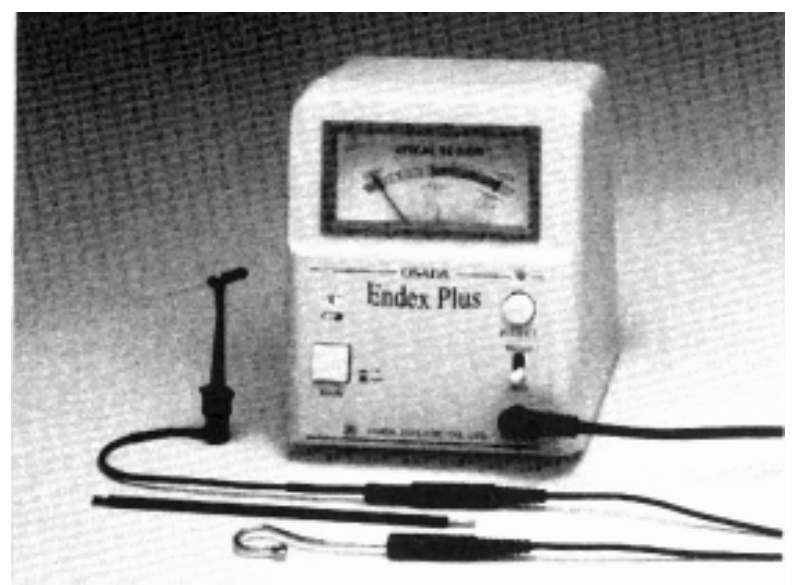

Figura 4. Localizador Apical Endex Plus. Imagen comercial. Journal of Endodontics Vol. 28 № 3, Marzo de 2002
Actualmente. se encuentran el localizador de ápices Neosono Última EZ (Amadent, Cherry Hill, NJ, USA) y el último modelo Neosono Co-Pilot (Figura 5). Además puede ser usado como vitalómetro.

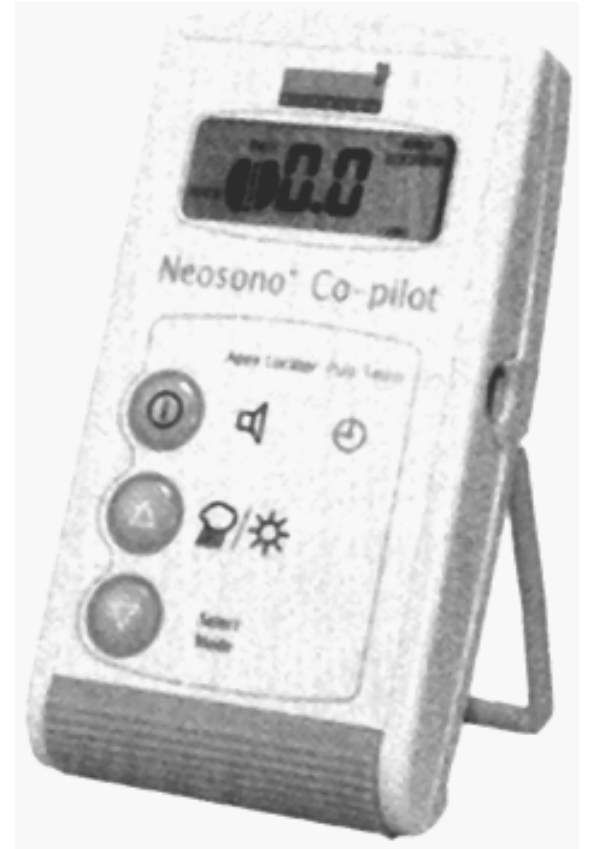

Figura 5. Localizador apical Neosono Co-Pilot. Imagen comercial. Journal of Endodontics Vol. $27 \mathrm{~N}^{\circ}$ 6, Junio de 2001

Recientemente salieron al mercado dos nuevos localizadores apicales producidos por Analytic, Sybron Dental Specialities, distribuidos por Kerr Dental Mfg., el Apex Finder 7001 y Endo Analyzer 8001.

El sistema de localización apical de estos dos aparatos es igual, localiza el foramen apical monitoreando la impedancia entre dos electrodos, solamente difieren en que el Endo Analyzer 8001 trae incorporado una vítalo metro. ${ }^{3}$

Un estudio del 2000, comparó el Neosono Última EZ, el Apit y el Apex-Finder. Se demostró que todos fueron capaces de dar lecturas precisas en presencia de humedad, pero el más preciso fue el localizador Apex Finder AFA Modelo 7005. ${ }^{24}$

\section{Tri-Auto ZX (J.Morita Co., Kyoto, Japan).}

Recientemente se introdujo en el mercado una innovadora pieza de mano diseñada para la instrumentación mecánica del conducto radicular, que, además, puede usarse como medidor electrónico de la longitud del conducto 


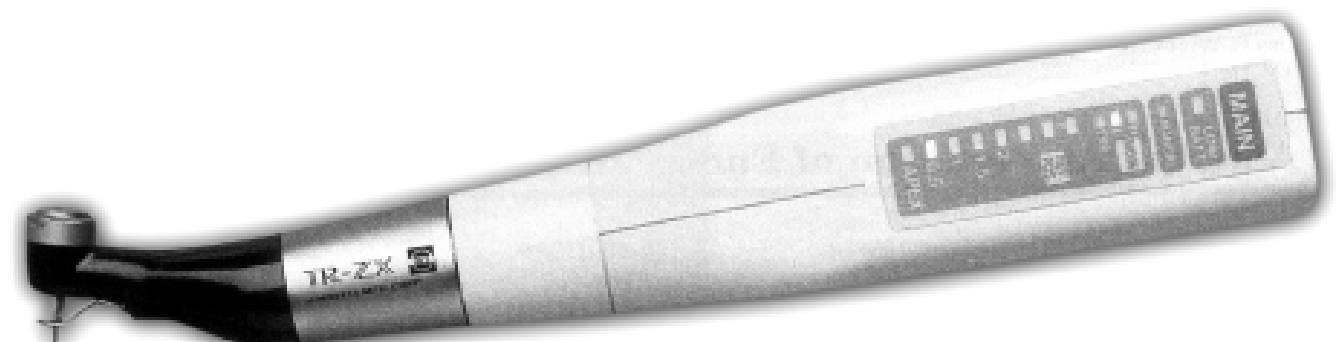

Figura 6. Localizador Apical Tri-Auto ZX. Imagen comercial. Journal of Endodontics Vol. 28 № 3, Marzo de 2002

radicular: Tri-Auto ZX (J. Morita Co., Kyoto, Japan). (Figura 6) Durante la instrumentación, la constricción apical es frecuentemente ensanchada. ${ }^{25}$ Park, registra que esto se produce en el 55,9\% de los casos. ${ }^{26}$

Aunque la endodoncia es un tratamiento secuencial y sistematizado, siendo muy importante respetar todos los pasos terapéuticos necesarios, se sabe que la clave radica en una limpieza y desinfección efectiva de los canales radiculares. El sistema Endox (Lysis Deutschland GMBH) .

Este sistema permite mediante el uso de una corriente alterna de alta frecuencia, (600 Khz. por 1/10 seg.) la vaporización del tejido pulpary del contenido bacteriano. Además, lleva incorporado un sistema para localizar el ápice, con el fin de mejorar la calidad del tratamiento endodóntico y de simplificar la sistematización del trabajo. ${ }^{27}$

Inicialmente localiza el ápice del conducto y posteriormente vaporiza la pulpa (paquete neurovascular, componente celular y sistema conectivo) en forma instantánea y sencilla; elimina el tejido pulpar y los microorganismos del sistema de los conductos radiculares.

Así mismo, permite una remoción más conservadora de la dentina del conducto radicular y el uso de una menor cantidad de sustancia irrigante en comparación con un tratamiento de endodoncia convencional. Es menos doloroso, pues como es bien sabido, el dolor postoperatorio se produce como resultado de la manipulación, el uso de irrigantes, la extrusión del material de relleno y las fracturas verticales, entre otros. Durante el estudio de Chaparro no se observaron diferencias postoperatorias entre los casos realizados en una misma sesión y los realizados en dos sesiones. No fue necesaria la prescripción de antibióticos y/o antinflamatorios y no se reportó ninguna complicación. ${ }^{27}$
Otro factor importante en el uso de este nuevo sistema es la significativa reducción del tiempo de trabajo, en el tratamiento endodóntico en comparación con el tratamiento convencional, beneficiando no sólo al paciente sino también al operador.

El pronóstico es bueno tanto para los dientes vitales como para los necróticos 0 con periodontitis apicales. ${ }^{27}$

\section{CONCLUSIONES}

Se puede concluir que el uso de localizadores de tercera generación es un método rápido, objetivo, cómodo y preciso para localizar la longitud de trabajo, evitando un mayor riesgo de radiación. Su uso frecuente permite al clínico adquirir una mayor destreza en su manipulación, lo que le permitirá obtener resultados mucho más precisos.

El uso de localizadores apicales no sustituye el método radiográfico; por el contrario, se complementan, porque la radiografia es necesaria para controlar las fases posteriores del tratamiento de endodoncia, así como es de gran utilidad en aquellos casos en los que es imposible utilizar el localizador.

A pesar de los grandes avances tecnológicos, existen algunas contraindicaciones que no han podido ser superadas como en los conductos no permeables, imposibilidad de aislar el conducto de la encía o de las restauraciones metálicas y su empleo en pacientes con marcapasos. Respecto de los últimos avances, son necesarios más estudios, para corroborar o desmentir la verdadera efectividad, que las diferentes casas comerciales les atribuyen.

40 


\section{BIBLIOGRAFÍA}

1. West J, Roane J. Sistema de limpieza y conformación de los canales radiculares. Cohen S., Burns R. Vías de la Pulpa. Séptima Edición. Madrid. Editorial Harcourt - Mosby. 1999. 209 - 211

2. Walton - Torabinejad. Endodoncia principios y Práctica. McGrawHill. Segunda Edición. México 1996. 211-21,.558

3. Rivas Muñoz R. Uso y eficacia del localizador del foramen fisiológico, Apex Finder 7001. URL: http://www.iztacala.unam.mx/ rrivas/ apexfinder.html

4. Pratten DH, McDonald NJ. Comparison of radiographic and electronic working lengths. J Endod 1996; 22: 173 - 176.

5. McDonald NJ, Hovland EJ. An evaluation of the apex locator endocater. J Endod 1990; 16: 5 - 8.

6. Ounsi HF, Haddad G. In vitro evaluation of the reliability of de Endex electronic Apex locator. J Endod 1998; 24: 120 - 121.

7. Kobayashi C. Electronic canal length measurement. Oral Surg Oral Med Oral Pathol 1995; 79: 226 - 231.

8. Kobayashi C. New electronic canal measuring deveice based on the ratio method. J Endod 1994; 20: 111 - 114.

9. Oishi A, Yoshioka T, Kobayashi C, Suda H. Electronic detection of root canal constriction J Endod 2002; 28: 361 - 364.

10. Hembrough J, Weine F, Pisano J., Eskoz N. Accuracy of an electronic apex locator: a clinical evaluation in maxillary molars. J Endod. 1993; 19: 242 - 246

11. Ibarrola JL, Chapman B, Howard JH, Knowles KI, Ludlow MO. Effect of prefaring on Root ZX apex locators. J Endod 1999; 25: 625 - 626.

12. Jenkins $\mathrm{W}$, Schindler $\mathrm{F}$. An in vitro evaluation of the accuracy of the root ZX in presence of various irrigants. J Endod 2001; 27: 209 - 211.

13. Frank AL, Torabinejad M. An In vivo evaluation of endex apex locator. J Endod 1993; 19: 177-179.

14. Bramamnte C. A critical evaluation of some methods of determining tooth length. Oral Surg Oral Med Oral Pathol 1974; 37 : 463469.

15. Shabahang S, Goon WY, Gluxkin AH. An in vivo evaluation of root ZX electronic apex locator. J Endod 1996; 22: 616 - 617.

16. Keller M, Brown C, Newton C. A clinical evaluation of endocater. An electronic apex locator J Endod 1991; 17: 271 - 274.

17. Czerw RJ, Fulkerson M, Donnelly JC, Walman JO. In Vitro Evaluation of the Accuracy of Several Electronic Apex Locators. J Endod 1995; 21: 572575

18. Pommer 0, Stamm. 0, Attin T. Influence of the canal contents on the electrical assisted determination of Length of Root Canals. J Endod 2002; $28: 83-85$
19. Pilot TF, Pitts DL. Determination of impedance changes at varying frequencies in relation to root canal position and irrigant.. JEndod1997; 23: 719 - 723

20. Cemal Tinaz A, Sibel Sevimli L, Güliz G, Emin G. The effects of sodium hypochlorite concentrations on the accuracy of an apex locating device. J Endod 2002; 28:160 162.

21.Beach CW , Douglas J, Jeffrey W. Use of an electronic apex locator on a cardiac pacemaker patient. J Endod 1996; 22: 182184.

22. Weiger R, John X, Geigle H y Löst C. An in vitro comparison of two modern apex locators. J Endod 1999; 25: 765-68.

23. Pagavino G, Pace R, Baccetti T. A SEM study of in vivo accuracy of the root ZX electronic apex locator. J Endod 1998; 24: 438-441.

24. Briceño B, Hähnel P, Willersheusen . Reliability of electronic apex locator to determine the working length under moisture conditions. J Endod 2000; 26: 551.

25. Campbell D, Friedman S, Nguyen HQ, Kaufman A, Keila S. Apical extent of rotary canal instrumentation with an apex- locating hand piece en vitro. Oral Surg Oral Med Oral, Pathol Oral Radio 1998; 85: 319- 324.

26. Park JW. Tri Auto ZX in locating apical foramen with rotary. (Abstract) J Endod 2000; 26: 561

27. Chaparro A, Murillo del Castillo C, Haffner C, Benz C, Hickel R. Sistema Endox: desvitalización y esterilización electrónica. Estudio clínico. URL: http://www.ueda.es/articulos/Art\%20avances.htm 